$\underline{\underline{\text { IIIIIII }}} \stackrel{\text { Open Access Articles }}{\text { Open }}$

\author{
Waveguide-integrated photonic crystal \\ spectrometer with camera readout
}

The MIT Faculty has made this article openly available. Please share how this access benefits you. Your story matters.

\begin{tabular}{|l|l|}
\hline Citation & $\begin{array}{l}\text { Meng, F., et al. "Waveguide-Integrated Photonic Crystal } \\
\text { Spectrometer with Camera Readout." Applied Physics Letters } 1055 \\
(2014) .\end{array}$ \\
\hline As Published & $10.1063 / 1.4892265$ \\
\hline Publisher & AlP Publishing \\
\hline \hline Version & Final published version \\
\hline Citable link & https://hdl.handle.net/1721.1/134238 \\
\hline Terms of Use & $\begin{array}{l}\text { Article is made available in accordance with the publisher's } \\
\text { policy and may be subject to US copyright law. Please refer to the } \\
\text { publisher's site for terms of use. }\end{array}$ \\
\hline
\end{tabular}




\section{AIP / Applied Physics Letters}

\section{Waveguide-integrated photonic crystal spectrometer with camera readout}

Fan Meng, Ren-Jye Shiue, Noel Wan, Luozhou Li, Jing Nie, Nicholas C. Harris, Edward H. Chen, Tim Schröder, Nadia Pervez, loannis Kymissis, and Dirk Englund

Citation: Applied Physics Letters 105, 051103 (2014); doi: 10.1063/1.4892265

View online: http://dx.doi.org/10.1063/1.4892265

View Table of Contents: http://scitation.aip.org/content/aip/journal/apl/105/5?ver=pdfcov

Published by the AIP Publishing

\section{Articles you may be interested in}

Array integration of thousands of photonic crystal nanolasers

Appl. Phys. Lett. 104, 121108 (2014); 10.1063/1.4869753

Waveguide photon-number-resolving detectors for quantum photonic integrated circuits Appl. Phys. Lett. 103, 111116 (2013); 10.1063/1.4820842

Efficient quantum dot single photon extraction into an optical fiber using a nanophotonic directional coupler Appl. Phys. Lett. 99, 121101 (2011); 10.1063/1.3617472

Butt-end fiber coupling to a surface-emitting $\Gamma$-point photonic crystal band edge laser Appl. Phys. Lett. 90, 171115 (2007); 10.1063/1.2732835

All-optical bistable switching in curved microfiber-coupled photonic crystal resonators Appl. Phys. Lett. 90, 161118 (2007); 10.1063/1.2724921

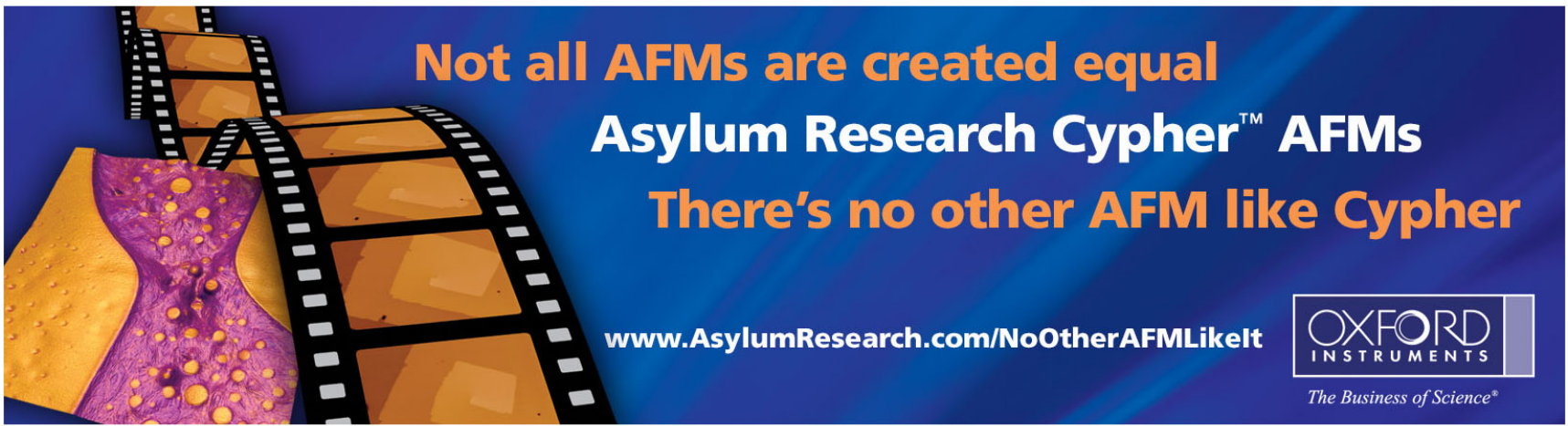




\title{
Waveguide-integrated photonic crystal spectrometer with camera readout
}

\author{
Fan Meng, ${ }^{1}$ Ren-Jye Shiue, ${ }^{1}$ Noel Wan, ${ }^{2}$ Luozhou Li, ${ }^{1}$ Jing Nie, ${ }^{1}$ Nicholas C. Harris, ${ }^{1}$ \\ Edward H. Chen, ${ }^{1}$ Tim Schröder, ${ }^{1}$ Nadia Pervez, ${ }^{3}$ loannis Kymissis, ${ }^{3,4}$ and Dirk Englund ${ }^{1, a)}$ \\ ${ }^{1}$ Department of Electrical Engineering and Computer Science, Massachusetts Institute of Technology (MIT), \\ Cambridge, Massachusetts 02139, USA \\ ${ }^{2}$ Department of Physics, Columbia University, New York, New York 10027, USA \\ ${ }^{3}$ Chromation Partners LLC, 18 Bridge Street Suite 2J, Brooklyn, New York 11201, USA \\ ${ }^{4}$ Department of Electrical Engineering, Columbia University, New York, New York 10027, USA
}

(Received 29 March 2014; accepted 24 July 2014; published online 4 August 2014)

\begin{abstract}
We demonstrate an infrared spectrometer based on waveguide-coupled nanocavity filters in a planar photonic crystal structure. The input light is coupled into the waveguide, from which spectral components are dropped into the cavities and radiated off-chip for detection on a commercial InGaAs camera. The spectrometer has a footprint of only $60 \mu \mathrm{m}$ by $8 \mu \mathrm{m}$. The spectral resolution is about $1 \mathrm{~nm}$ in the operation bandwidth of $1522-1545 \mathrm{~nm}$. By substituting the membrane material and structure parameters, this design can be easily extended into the visible regime and developed for a variety of highly efficient, miniature photonic applications. ( 2014 AIP Publishing LLC.

[http://dx.doi.org/10.1063/1.4892265]
\end{abstract}

Motivated by the need for compact, high-performance spectrographs, a wide variety of chip-integrated devices have been investigated, including microresonator arrays, ${ }^{1,2}$ photonic crystals $(\mathrm{PhCs}),{ }^{3-5}$ and arrayed waveguide gratings (AWGs). ${ }^{6}$ For certain applications such as biochemical and environmental sensing, ${ }^{7,8}$ there has been an interest to develop planar spectrometers that scatter dispersed light out of the chip so that it can be imaged using simple imaging arrays such as cameras. Pervez et al. ${ }^{4}$ demonstrated a PhC grating pattern integrated into a slab waveguide, which could be imaged using a camera. The spectral resolution of this device reached $\Delta \lambda / \lambda \sim 0.1$ in the visible spectrum, where $\Delta \lambda$ is spectral resolution and $\lambda$ is the central wavelength of operating range. Gan et $a l^{3}$ addressed the spectral resolution problem by replacing the grating with an array of photonic crystal nanocavities that coupled light from the planar waveguide out of the plane into a camera. This approach improved the spectral resolution to $\Delta \lambda / \lambda \sim 4 \times 10^{-4}\left(\sim 1 \times 10^{-4}\right)$ in the visible (near-infrared) spectrum. However, both approaches suffered from a low efficiency since the incident photon flux was spread nearly uniformly across all spectral filters and each filter only scattered up a small portion of the spectrum towards the camera. In a linear waveguide, the photon flux can be guided along a series of spectral filters, so that every photon can, in principle, be directed into the spectrally matched output port. Thus, linear waveguides have been the basis of numerous in-plane spectral filters for wavelength detection.

Here, we demonstrate a spectrograph relying on a linear waveguide coupled to an array of cavities that direct the filtered field upward for detection with a camera, which greatly simplifies the mode of operation than its counterparts. ${ }^{9,10}$ This design provides both off-chip detection with standard cameras, high spectral resolution enabled by the cavity design, and high photon efficiency. Our device employs a $\mathrm{PhC}$ cavity array side-coupled to a line-defect waveguide with a total device efficiency of $6.36 \%$ and a spectral

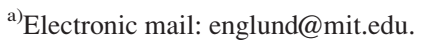

resolution of $\Delta \lambda / \lambda \sim 6.5 \times 10^{-4}$. The device operates at a wavelength near $1550 \mathrm{~nm}$ with a bandwidth of $24 \mathrm{~nm}$. By extending the number of nanocavities whose resonances fall into the bandgap of the line-defect waveguide, this type of waveguide-integrated spectrometer could allow hundreds of wavelength channels to be simultaneously monitored using an off-the-shelf camera.

As the scanning electron microscope (SEM) image shown in Fig. 1(a), the micro-scale spectrometer device consists of a line-defect waveguide in a triangular planar $\mathrm{PhC}$ lattice, side-coupled to 24 linear three-hole defect (L3) nanocavities. ${ }^{11}$ The indices of $\mathrm{C} 1-\mathrm{C} 24$ are marked for each cavity and the cavity resonant modes are tuned by shifting of the end-holes of the L3 design: $\Delta s=0.15 a+0.005 a *(n-1)$, where $a$ is the lattice constant and $n$ is the cavity index. The device was fabricated on a silicon-on-insulator (SOI) wafer with $200 \mathrm{~nm}$ thick silicon top membrane. The PhC structures are formed by a series of electron-beam lithography (EBL), dry etching, and wet chemical (hydrofluoric acid) undercutting of $\mathrm{SiO}_{2}$ sacrificial layer under the membrane. The lattice constant of $a=440 \mathrm{~nm}$ and the hole radius $r=0.29 a$ were chosen to achieve a bandgap within the telecom band. To get a better bandgap overlapping between the line-defect waveguide and L3 cavities, two rows of the holes next to the waveguide were outwards shifted from the waveguide by $0.03 a$, respectively. All cavities were $60^{\circ}$ rotated and spaced by three lattices with respect to the waveguide axis to improve the coupling efficiency. ${ }^{12}$ Meanwhile, we also introduced a series of perturbed holes in the vicinity of the cavity defect. These perturbed holes serve to shape the far-field radiation of the cavity modes to match a free space Gaussian mode, therefore increasing the out coupling of the cavities, ${ }^{13}$ as shown in the inset of Fig. 1(a). The resonant wavelengths of the cavities were gradually red-shifted by progressively shifting the L3 cavity end-holes outwards, achieving channel spacing of $\sim 1 \mathrm{~nm}(\sim 128 \mathrm{GHz})^{11}$ for 24 cavities to span an operating range from 1522 to $1545 \mathrm{~nm}$-well within the $\mathrm{PhC}$ lattice bandgap. Shown in Figs. 1(b)-1(d) are simulations of 


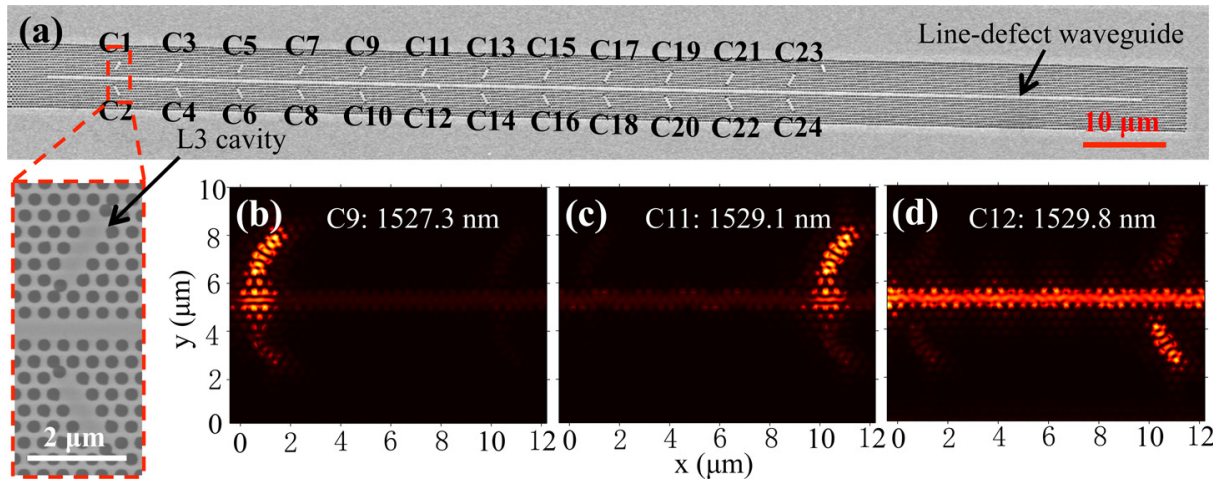

FIG. 1. (a) SEM image of the fabricated photonic crystal (PhC) chip, showing the $24 \mathrm{~L} 3$ nanocavities $60^{\circ}$ tilted relative to the axis of the line-defect waveguide. All the cavities distribute in pairs to form 12 periods. Scale bar: $10 \mu \mathrm{m}$. Inset: magnified image of one period, a higher coupling efficiency between the line-defect waveguide and L3 cavities was realized by this configuration. (b)-(d) Simulated electric field intensity $\left(|E|^{2}\right)$ distribution for three L3 nanocavities (C9, C11, and C12) excited by the line-defect waveguide's fundamental mode and the corresponding resonant wavelengths are $1527.3 \mathrm{~nm}$ for C9, $1529.1 \mathrm{~nm}$ for $\mathrm{C} 11$, and $1529.8 \mathrm{~nm}$ for $\mathrm{C} 12$, respectively.

the electric field intensities $|E|^{2}$ in a short device section containing three cavities $(\mathrm{C} 9, \mathrm{C} 11$, and $\mathrm{C} 12)$ with resonant modes located across the spectrometer's spectrum at $1527.3 \mathrm{~nm}, 1529.1 \mathrm{~nm}$, and $1529.8 \mathrm{~nm}$, respectively; the full 24-cavity spectrometer devices was not simulated as a whole because of memory limitation to 16 GB. In these simulations, we first considered the fundamental line-defect waveguide mode at 1520 through $1550 \mathrm{~nm}$ in increments of $5 \mathrm{~nm}$ using an eigenmode solver, then propagated these modes through the waveguide-cavity section using finite-difference time-domain (FDTD) software. Because the modal dispersion in the range from 1520 to $1550 \mathrm{~nm}$ is small, with an index difference of 0.04 for the fundamental mode, we found it adequate to neglect differences in modal profile over the $5 \mathrm{~nm}$ windows. These simulations clearly show the wavelength-dependent excitation and outcoupling through the cavity modes, as expected. With optimized fabrication and pattern parameters, higher $Q$ factors can be reached, resulting in a higher spectral resolution below $0.1 \mathrm{~nm} .^{9,11,14}$ Also, with precise tuning of lattice constant and a larger number of $\mathrm{L} 3$ nanocavities, this $\mathrm{PhC}$ structure can realize a controllable channel spacing and operation bandwidth.

The probe light is introduced into the line-defect waveguide through a coupling method (fiber taper coupling in this paper). The waveguided light then couples into the $24 \mathrm{PhC}$ nanocavities and transmits out of plane selectively at corresponding resonant wavelengths. Sweeping through the resonant wavelengths of all these cavities, the light is dispersed and filtered into a two-dimensional (2D) detector array. Monitoring the scattered light from each spatial position provides the spectral content of the input light, as shown in Fig. 2(a). We fabricated the fiber taper by pulling an infrared (IR) single mode fiber over a hydrogen flame. A controlled-stretch across $26 \mathrm{~mm}$ over a heat zone of $10 \mathrm{~mm}$ produced a fiber taper with diameter of $1 \mu \mathrm{m}$. The fiber taper is then precisely positioned on top of the line-defect waveguide. A polarization controller (PC) is used to optimize the coupling between the fiber and the TE mode of the PhC waveguide. The scattered light from each nanocavity is collected by an objective lens $(\mathrm{NA}=0.55)$ and directed to either the $0.5 \mathrm{~m}$ long commercial spectrometer or an uncooled InGaAs camera (IRVista). The experimental setup for this demonstration is shown in Fig. 2(b). In Fig. 2(c), an optical image shows a fiber taper coupled with the line-defect waveguide of this device (\#3: from top to bottom). A yellow box highlights the section where the fiber and waveguide are positioned together.

When the tapered fiber is optimally positioned on the $\mathrm{PhC}$ waveguide, we observe a marked drop in transmission for spectral components resonant with waveguide modes, as seen in Fig. 3(a). We infer from the transmission before and after placing the fiber on the waveguide that the fiber-to-waveguide (a)

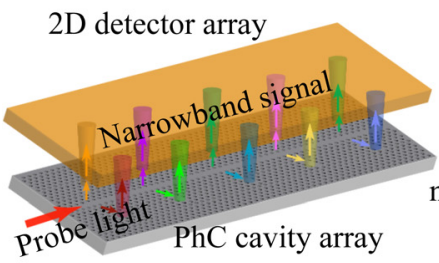

(b)

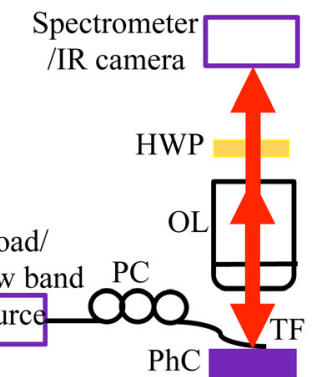

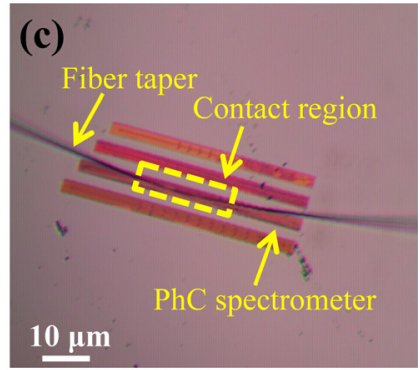

FIG. 2. (a) Schematic of the compact spectrometer based on L3 PhC nanocavities coupled with a line-defect waveguide, with the probe light coupled into the waveguide and decoded into many narrowband signals by cavity modes. (b) Confocal microscope setup used to characterize the device via the scattered signals from nanocavities. A broad or narrowband laser is coupled into the device and filtered by the cavities into narrowband (resonant) signals, which are detected by a commercial spectrometer or a Vista IR camera. HWP: half-wave plate, OL: objective lens, TF: tapered fiber, PC: polarization controller. (c) Optical image of the working device: a fiber taper coupled with the line-defect waveguide. Scale bar: $10 \mu \mathrm{m}$. 
(a)

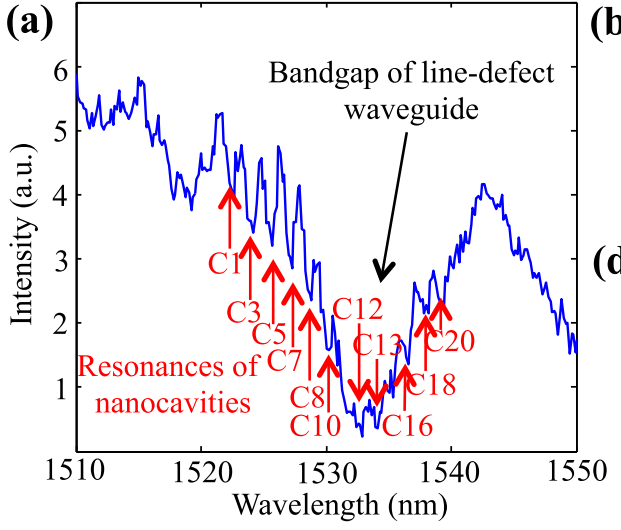

coupling efficiency $\left(\eta_{1}\right)$ is $68 \%$. The broad transmission drop from 1520 to $1545 \mathrm{~nm}$ corresponds to coupling to the $\mathrm{PhC}$ line-defect waveguide, while the multiple narrow dips correspond to additional loss via the waveguide-coupled nanocavities, as are also marked in the spectrum. After this broadband characterization, we switched the input light source to a tunable narrowband laser $(5.6 \mathrm{fm}$ linewidth) and recorded the cavity emission as a series of images on the IR camera. Figs. 3(c)-3(e) show spatially resolved images of three cavities (C5, C6, and C7) obtained when the laser was tuned to their resonant wavelengths of $1525.79 \mathrm{~nm}$, $1527.28 \mathrm{~nm}$, and $1528.15 \mathrm{~nm}$, respectively. These corresponding cavities are indicated in the SEM image in Fig. 3(b). In each camera image, a bright spot with high contrast from other cavity regions comes from matching between the narrowband light and cavity resonance, which illustrates the function of the cavities as wavelength-selective channels. These sweeping results of narrowband light source demonstrate that the cavity array spectrometer enables spectral resolution equal to the bandwidth of the cavity modes. To obtain the spectrum of an unknown input field from such images, a calibration of the cavity emission to spectral components is required. In practical applications, it is necessary to attach the tapered fiber with the chip and make them a monolithic package. Our fiber taper is manipulated in ambient environment and is placed in contact with the silicon planar $\mathrm{PhC}$ waveguide. The contact of the tapered fiber is held

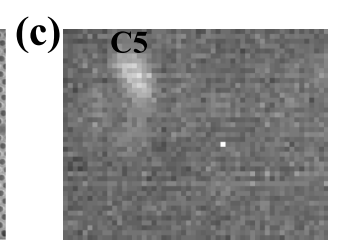

(e)

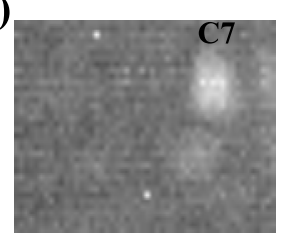

FIG. 3. (a) Transmission spectrum of the tapered fiber when coupled with the device. The broad dip results from the bandgap of the line-defect waveguide; the series of small dips are due to coupling into the cavity modes. (b) SEM image of one section of the device, scale bar: $2 \mu \mathrm{m}$. (c)-(e) Optical images of cavity transmission gotten from a narrowband laser and IR camera configuration: the laser wavelengths coincide with the resonances of C5, C6, and C7 cavities, respectively.

in place by surface forces. For packaging, the interface can be made robust by gluing the tapered fiber directly on the chip. In our current configuration, we have achieved a maximum of nearly $70 \%$ coupling efficiency from fiber to $\mathrm{PhC}$ waveguide. The efficiency can be further improved by engineering the diameter of the fibered taper for mode-matching with the photonic crystal waveguide. Alternatively, if the $\mathrm{PhC}$ waveguide is coupled with an on-chip silicon waveguide and an edge coupler, the off-chip coupling can be realized using only a single glued edge-coupling fiber (with coupling efficiency of $80 \%{ }^{15}$ ). It is now commercially possible to couple to silicon photonic circuits with sub-dB losses using edge coupling or grating coupling. These couplers can be bonded into place to allow for stable operation of the device.

The spectral calibration can be obtained in two different configurations, which are illustrated in Fig. 4(a) for one of the cavities, C6. The top panel of Fig. 4(a) plots the reflection spectrum of the cavity (red line), which is obtained in a cross-polarized configuration ${ }^{16}$ by illuminating the cavity vertically from a free space objective with a broadband laser $(0.95-2 \mu \mathrm{m})$ and measuring the reflection with a commercial IR spectrometer. This measurement indicates a $Q$ factor of 1950. The transmission spectrum of the same cavity is obtained by exciting the cavity with a tunable narrowband $(\sim \mathrm{MHz})$ laser source through the tapered fiber, $\mathrm{PhC}$ waveguide, and the cavity. The intensity of scattered light from
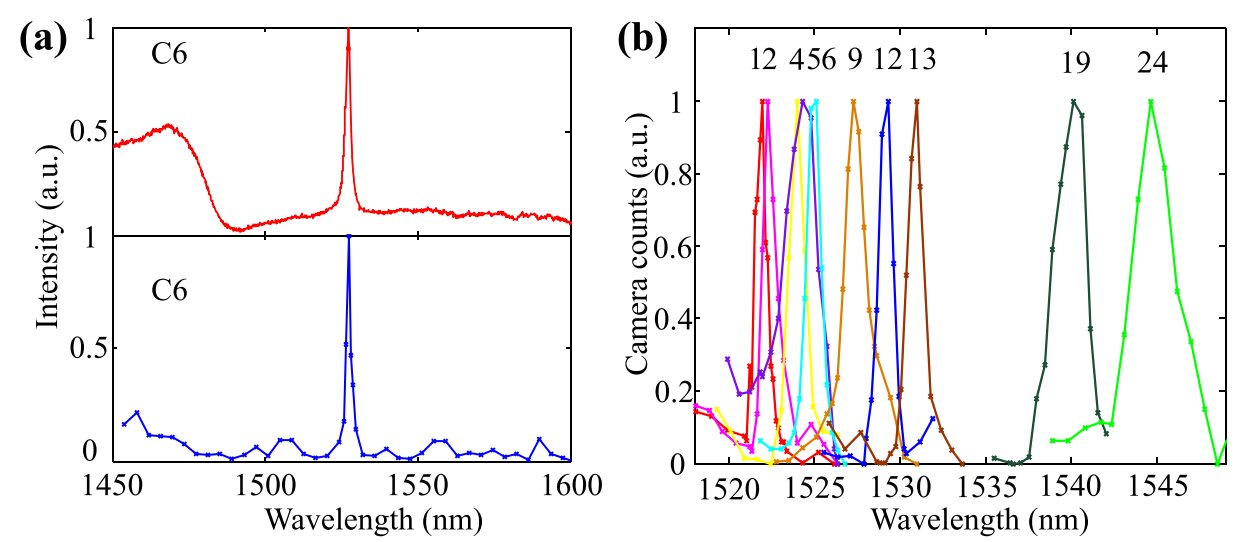

FIG. 4. (a) Reflection (red) and transmission (blue) spectra of C6 showing a resonance at $1527.28 \mathrm{~nm}$. The reflection is obtained directly from the commercial spectrometer and the transmission spectrum is obtained by integrating the counts across the cavity region at multiple wavelengths, showing a good agreement. (b) Transmission spectra of the ten nanocavities (C1 $(Q=1834.2), \mathrm{C} 2$ ( $Q=1958.7), \mathrm{C} 4(Q=1366.3)), \mathrm{C} 5(Q=476.5), \mathrm{C} 6(Q=1868.9), \mathrm{C} 9(Q=766.2), \mathrm{C} 12$ $(Q=1948.7), \mathrm{C} 13(Q=642.3), \mathrm{C} 19 Q=(570.1), \mathrm{C} 24(Q=562.1))$ from the IR camera readout. 
the cavity is collected using the IR camera. The resulting transmission spectrum is plotted in the bottom panel of Fig. 4(a), matching the reflectivity measurement in the top panel. Thus, an input spectrum can be measured by projection onto the drop cavities, which is detected using an off-chip camera. To make a general purpose spectrometer, the resonant wavelengths of the cavities would distribute uniformly and cover the whole spectrum of the target source. The spectra of ten of the L3 nanocavities are also sampled and summarized for a wider range of wavelengths, as shown in Fig. 4(b), indicating a linewidth of $\Delta \lambda \sim 1 \mathrm{~nm}$. The gradual $Q$ drop of these cavities results from the increment of end-hole shifting from $\mathrm{C} 1$ to $\mathrm{C} 24$. This tuning mechanism unfortunately also changes the quality factor. To avoid this, a different tuning mechanism, e.g., incrementing the air hole diameters could be used to blue-shift cavities. ${ }^{17}$

The coupling of the cavities to the waveguide was designed to be in the critical coupling regime since this optimizes the efficiency. Using the measured cavity quality factors, we can estimate the experimental coupling coefficients using coupled mode theory. ${ }^{18}$ In the waveguidecavity system, the side-coupled waveguide is treated as a perturbation for individual L3 cavities. We measured that the resonant mode of an unloaded cavity (far away from the waveguide) with optimal spacing of $\Delta s=0.15 a$ occurs at $\lambda_{0}=1525.8 \mathrm{~nm}$ with $Q_{0}=1948.7$. This quality factor is limited by scattering, not material losses. When this cavity is coupled to the waveguide at a distance of $3 a$, we measured a drop in the quality factor to $Q^{\prime}=778.9$ and a wavelength red-shift by $\Delta \lambda=1.48 \mathrm{~nm}$ to $\lambda^{\prime}=1527.28 \mathrm{~nm}$. The coupling efficiency from waveguide mode to free space mode through the cavity, $\eta_{2}$, is estimated from coupled mode theory as $4 \kappa_{w} \eta_{c-o b j} \kappa_{c} /\left(\kappa_{c}+\kappa_{w}\right)^{2} \approx 9.35 \%,{ }^{18,19}$ where the cavity intrinsic loss $\kappa_{c}=\omega_{0} / Q_{0}$ is $0.63 \mathrm{THz}$, the loss rate into the waveguide $\kappa_{w}=\omega^{\prime} / Q^{\prime}-\omega_{0} / Q_{0}$ is $0.95 \mathrm{THz}$, and $\eta_{c-o b j}$ is the coupling efficiency of the cavity mode into the imaging objective mode. From the far-field emission of the L3 mode profile, $\eta_{c-o b j}$ is calculated by the integral over the sub-critical solid angle defined by the NA of the objective lens, yielding $\eta_{c-o b j} \sim 10 \% .^{20,21}$ This coupling efficiency of the cavity mode towards the objective can be increased by employing cavity designs with tailored radiation patterns. ${ }^{13,21}$ The total device efficiency $\eta_{1} \times \eta_{2} \approx 6.36 \%$. This efficiency could be further improved if the coupling from the tapered fiber to the PhC waveguide is optimized; nearly $100 \%$ coupling has been demonstrated previously using such a tapered fiber coupling approach. ${ }^{22}$ In addition, the coupling from the L3 cavity modes to a free space objective can be as high as $28 \%$ using an objective with NA $=0.75 .{ }^{21}$ With these optimizations, the overall efficiency of our device could be as high as $28 \%$, which is with a factor of two of a commercial grating-based spectrometer. Because of the broad transmission range of $\mathrm{Si}$, this spectroscopy can be extended to longer wavelengths into the mid-infrared.

In conclusion, we have demonstrated a compact, flexible, and economical spectrometer based on 24 L3 nanocavities side-coupled with a PhC line-defect waveguide. Spectral components of waveguide-coupled input light are filtered through these cavities and emitted to a camera for detection. Using a tapered fiber to introduce light into the line-defect waveguide, $6.36 \%$ device efficiency is obtained. Within the $1520-1545 \mathrm{~nm}$ range, spectral resolution of $1 \mathrm{~nm}$ at $1527 \mathrm{~nm}$ was realized at a footprint of $60 \mu \mathrm{m}$ by $8 \mu \mathrm{m}$. With the improvement of fabrication process and structural parameters, higher resolution, uniform channel spacing, and broader operation bandwidth are possible. Integrated with the same detector array and calibrated from spatial to spectral mapping, this packaged on-chip spectrometer can find a wide application in spectroscopy analysis.

This material was based upon work supported by the United States Air Force under Contract No. FA9101-12-C0010, under subcontract from Chromation Partners LLC. E. $\mathrm{H}$. Chen was supported by the NASA Office of the Chief Technologist Space Technology Research Fellowship. T. Schröder was supported by the Alexander von Humboldt Foundation.

${ }^{1}$ B. B. C. Kyotoku, L. Chen, and M. Lipson, "Sub-nm resolution cavity enhanced micro-spectrometer," Opt. Express 18, 102-107 (2010).

${ }^{2}$ Z. Xia, A. A. Eftekhar, M. Soltani, B. Momeni, Q. Li, M. Chamanzar, S. Yegnanarayanan, and A. Adibi, "High resolution on-chip spectroscopy based on miniaturized microdonut resonators," Opt. Express 19, 12356-12364 (2011).

${ }^{3}$ X. Gan, N. Pervez, I. Kymissis, F. Hatami, and D. Englund, "A highresolution spectrometer based on a compact planar two dimensional photonic crystal cavity array," Appl. Phys. Lett. 100, 231104 (2012).

${ }^{4}$ N. K. Pervez, W. Cheng, Z. Jia, M. P. Cox, H. M. Edrees, and I. Kymissis, "Photonic crystal spectrometer," Opt. Express 18, 8277-8285 (2010).

${ }^{5}$ B. Momeni, E. S. Hosseini, and A. Adibi, "Planar photonic crystal microspectrometers in silicon-nitride for the visible range," Opt. Express 17, 17060-17069 (2009).

${ }^{6}$ N. Ismail, L.-P. Choo-Smith, K. Wörhoff, A. Driessen, A. C. Baclig, P. J. Caspers, G. J. Puppels, R. M. de Ridder, and M. Pollnau, "Raman spectroscopy with an integrated arrayed-waveguide grating," Opt. Lett. 36, 4629-4631 (2011).

${ }^{7}$ M. N. Fiddler, I. Begashaw, M. A. Mickens, M. S. Collingwood, Z. Assefa, and S. Bililign, "Laser spectroscopy for atmospheric and environmental sensing," Sensors 9, 10447-10512 (2009).

${ }^{8}$ J. W. Chan, D. Motton, J. C. Rutledge, N. L. Keim, and T. Huser, "Raman spectroscopic analysis of biochemical changes in individual triglyceriderich lipoproteins in the pre- and postprandial state," Anal. Chem. 77, 5870-5876 (2005).

${ }^{9}$ H. Takano, B.-S. Song, T. Asano, and S. Noda, "Highly efficient multichannel drop filter in a two-dimensional hetero photonic crystal," Opt. Express 14, 3491-3496 (2006).

${ }^{10}$ S. Yamada, B.-S. Song, T. Asano, and S. Noda, "Silicon carbide-based photonic crystal nanocavities for ultra-broadband operation from infrared to visible wavelengths," Appl. Phys. Lett. 99, 201102 (2011).

${ }^{11} \mathrm{Y}$. Akahane, T. Asano, and B.-S. Song, "High- $Q$ photonic nanocavity in a two-dimensional photonic crystal," Nature 425, 944-947 (2003).

${ }^{12}$ A. Faraon, E. Waks, D. Englund, I. Fushman, and J. Vučković, "Efficient photonic crystal cavity-waveguide couplers," Appl. Phys. Lett. 90, 073102 (2007); e-print arXiv:physics/0610105v1 [arXiv:physics].

${ }^{13}$ C.-C. Tsai, J. Mower, and D. Englund, "Directional free-space coupling from photonic crystal waveguides," Opt. Express 19, 20586-20596 (2011).

${ }^{14}$ P. Rakich, H. Sotobayashi, J. Gopinath, S. Johnson, J. Sickler, C. Wong, J. Joannopoulos, and E. Ippen, "Nano-scale photonic crystal microcavity characterization with an all-fiber based 1.2 - 2.0 mum supercontinuum," Opt. Express 13, 821-825 (2005).

${ }^{15}$ A. Khilo, M. A. Popović, M. Araghchini, and F. X. Kärtner, "Efficient planar fiber-to-chip coupler based on two-stage adiabatic evolution," Opt. Express 18, 15790-15806 (2010).

${ }^{16}$ D. Englund, A. Faraon, I. Fushman, N. Stoltz, P. Petroff, and J. Vucković, "Controlling cavity reflectivity with a single quantum dot," Nature $\mathbf{4 5 0}$, 857-861 (2007).

${ }^{17}$ C. Kang, S. M. Weiss, Y. A. Vlasov, and S. Assefa, "Optimized lightmatter interaction and defect hole placement in photonic crystal cavity sensors," Opt. Lett. 37, 2850-2852 (2012). 
${ }^{18}$ X. Gan, K. F. Mak, Y. Gao, Y. You, F. Hatami, J. Hone, T. F. Heinz, and D. Englund, "Strong enhancement of light-matter interaction in graphene coupled to a photonic crystal nanocavity," Nano Lett. 12, 5626-5631 (2012).

${ }^{19}$ R.-J. Shiue, X. Gan, Y. Gao, L. Li, X. Yao, A. Szep, D. Walker, J. Hone, and D. Englund, "Enhanced photodetection in graphene-integrated photonic crystal cavity," Appl. Phys. Lett. 103, 241109 (2013).

${ }^{20}$ G. Shambat, Y. Gong, J. Lu, S. Yerci, R. Li, L. Dal Negro, and J. Vucković, "Coupled fiber taper extraction of 1.53 microm photoluminescence from erbium doped silicon nitride photonic crystal cavities," Opt. Express 18, 5964-5973 (2010).

${ }^{21}$ M. Toishi, D. Englund, A. Faraon, and J. Vucković, "High-brightness single photon source from a quantum dot in a directional-emission nanocavity," Opt. Express 17, 14618-14626 (2009).

${ }^{22}$ P. E. Barclay, K. Srinivasan, M. Borselli, and O. Painter, "Experimental demonstration of evanescent coupling from optical fiber tapers to photonic crystal waveguides," Electron Lett. 39, 842-844 (2003). 